\title{
The Drive for Integration: Some Comments
}

\author{
Hazel Markus \\ University of Michigan
}

Received September 25, 1980

In this article, Sanders has accomplished a thorough and impressive organization of the recent research that falls under the general rubric of "social facilitation." The paper is notably successful in its attempts to pull together a wide-ranging literature, in its delineation of some of the common patterns of findings assumed to support each theoretical alternative, and in its final suggestion that a synthesis of the various approaches to social facilitation may be necessary to explain the phenomenon fully. The article suffers, however, because Sanders apparently believes that a synthesis and integration of the various approaches to social facilitation can be best achieved by contrasting all approaches against the distraction/conflict model and then subsuming them under this view. Most of the article is devoted to various attempts at demonstrating the relatively greater validity of the distraction/conflict model. This results in a variety of difficulties including: (1) inappropriate comparisons, (2) substitution of description and labeling for explanation, and (3) the premature exclusion of some more simple or minimal explanations of social facilitation. Each of these points will be discussed briefly below, but together these difficulties work to undermine what should be a major purpose of this type of article-the illustration of the potential generality and importance of the social facilitation phenomenon to an understanding of social behavior.

With respect to the first point, I think it is questionable whether Sanders has accomplished an integration of the social facilitation research, as the title implies. Integration implies that there is conceptual and empirical similarity across the three approaches of mere presence, learned drive, and distraction/conflict. These approaches do indeed share the label "social facilitation," but this nominal similarity masks the fact that these are three very different levels of explanation aimed at explaining

Requests for reprints should be addressed to Dr. Hazel Markus, Institute for Social Research, University of Michigan, Ann Arbor. MI 48106. 
different aspects of the phenomenon. The categorization of the research in Table 1 and the accompanying figures indicating which theory has received the most confirmations generates a false contest among the three approaches. Within this group of experiments, a study which confirms one theory does not necessarily serve to call into question or disconfirm another theory. Alternatively, a failure to disconfirm one theory does little to bolster our faith in another. How, then, are we to evaluate the results that distraction/conflict has been confirmed $80 \%$ of the time and not disconfirmed at all?

Here we must remind ourselves that the presence of similar effects, in this case, social facilitation (enhanced or impaired task performance), does not allow us to assume that the same variables are necessarily mediating these effects. Indeed, in several places throughout the article, the author states that the theories are not mutually exclusive and that they vary tremendously in complexity. In the final analysis, however. Sanders ignores his own admonitions and an attempt is made, presumably for the sake of parsimony, to subsume both mere presence and learned drive under one theory based on distraction/conflict. The result is a complex, multifactor theory, which may be on target for some social situations but which is decidedly overdrawn for other elementary forms of social facilitation. In the approach to theory testing that is assumed here, the big sprawling theory is pitted against the little theory, the simple theory against the more complex one and all that matters is how many of the theoretical predictions are confirmed. When the scores are tallied. the distraction/conflict theory wins, but what have we learned about the phenomenon of social facilitation?

For example, the studies focusing on a mere presence explanation do not claim to have developed a hypothesis that can account for all classes of social facilitation. Instead mere presence was discussed as a sufficient condition for social facilitation. The studies were concerned with demonstrating "the enhancement of an organism's dominant responses by the simple physical presence of the species mate, independent of any informational or interactional influences the others may exert." This is precisely the point where the Sanders ${ }^{\circ}$ review begins; it is his definition of social facilitation. It is his purpose to specify just how the simple presence of others works to enhance the dominant response. He offers the distraction/conflict explanation. While Sanders' purpose is a useful and important one, it is quickly apparent why a comparison of mere presence and distraction/conflict is not particularly compelling. These two explanations are at different levels of the phenomenon. One is a general explanation for the social facilitation phenomenon-physical presence leads to dominant-response enhancement. The other accepts this relationship and then attempts to explain the mediating mechanisms. 
Contrasting the two explanations is like arguing that the apple did not fall to the ground because of gravity but because the stem broke.

If Sanders is to realize his goal of determining why or how drive induction takes place, the type of global comparison of outcomes performed here is not sufficient. What is needed instead is a careful task and response analysis so that it would be possible to determine which studies were really comparable. For example, the studies which claim to evaluate the mere presence hypothesis are quite disparate in their tasks, subject expectations, and response parameters. A close comparison of those studies in which the mere presence hypothesis is not confirmed indicates that they are studies in which there has not been a clear "alone" condition as a comparison or baseline. Without this baseline, it is difficult to estimate how much social facilitation has been achieved by the mere presence of another. In many of these studies the subject is well aware of the experimenter in the "alone" condition. Once the experiment's actual or implied presence is made salient to the subject, the task carries with it the promise of evaluation and gives rise to a variety of other outcomes such as competition, social comparison. and anxiety-factors that are quite likely to influence drive and thus to overshadow the effects of just mere presence. Those studies which Sanders claims actively disconfirm the mere presence hypotheses are similar in that they introduce the additional factor of stress into the experimental situation. Stressing the subject may immediately elevate drive to such a level that it is no longer possible to evaluate the contribution of the mere presence of a conspecific. The larger point is that a more precise comparison of studies and the conditions of these studies is necessary before particular theoretical alternatives are discarded.

Sanders' attempts to explain all social facilitation phenomena within one frame work lead to the second difficulty with this review articlethe substitution of elaborate labeling and description for explanation. The term "distraction/conflict" describes and labels what happens to an individual's attention in the presence of another, but it does not explain drive induction. Is it the case that the cockroach runs faster in a maze because of a compelling need to overcome the distraction produced by a species mate? The distraction/conflict term may aptly describe the cockroach's overt pattern of behavior, but does little to explain the general arousal or energizing of behavior produced by a conspecific. In fact, the primary contribution of the distraction/conflict hypothesis is to increase the assumed complexity of the phenomenon but it does not, in return, provide any greater a priori explanatory power. The theory is more specific with respect to what the subject might be doing in the presence of another but it is weak and lumpy when outlining the connection between distraction and response conflict and drive. With respect 
to enhancement of the dominant response on simple tasks, we find that it is trying to overcome distraction that explains the effect. With respect to difficult tasks. facilitation of the dominant response (impairment of performance) may be a function of a decrease in attention caused by the presence of others, or of trying too hard to overcome the distraction and doing poorly because the additional effort is facilitating the dominant (incorrect) response, or both.

The problem with the distraction/conflict hypothesis seems to rest with the concept of distraction. It is an ambiguous factor that has not been adequately conceptualized either here or in other work. Sanders, for example. ignores some relevant findings which suggest that distraction/ conflict and drive are not substitutable variables. The effects of distraction on behavior appear to habituate over trials while the effects of physical presence do not (cf. Zajonc. 1980). Again. it seems there is more to social facilitation than just distraction and response conflict. Also except for a few findings indicating that individuals feel more "distracted" in certain conditions, we have no evidence that what is labeled "distraction" is really distraction, no evidence that a division or conflict in response is actually taking place. In future studies, it would be useful to gather direct evidence about what the subject has attended to during the task. If we find social facilitation without evidence of attentional conflict we are left with the conclusion that it is not a necessary condition for the occurrence of socially induced drive. Alternatively, it may be found that attentional conflict does indeed occur but that it does not result in the predicted effects. We may see that what appears to result in overt response conflict in one aspect of the response may actually lead to improved performance on some other aspect of the response. Thus response latency in a task may be extended by the presence of another but memory or comprehension may be improved. This type of finding might lead us to consider more features of the response, to view social facilitation not as a unitary phenomenon-an all-or-none effect-but as a multicomponent process having a range of effects. Distraction/conflict may explain the initial phase of the response but some other concept may be needed to account for the energization of the response. Also, the effects of social facilitation may vary depending on the aspect of response (speed, accuracy, memory) under consideration.

A too hasty or uncritical acceptance of the distraction/conflict model also leads to the third difficulty-a tendency to reject prematurely some other more simple explanations of the phenomenon. Although the social facilitation process may be quite complex depending on circumstances and conditions, the mediating mechanisms need not be. For example. the mere presence explanation of social facilitation was meant to be just such a simple explanation. It was not meant as a complete explanation for all social facilitation phenomena, rather, the intent was to examine 
the minimal conditions under which an individual's dominant response would be enhanced by the physical presence of a conspecific. It is an explanation that emphasized, granted not in very specific terms, that some aspects of the social nature of individuals may indeed be instinctual or hard-wired and that this social nature or attitude may be stimulated by the presence of another member of the species. Whatever this mechanism was, it was thought to be simple, immediate in its effects, to consume only limited processing capacity, and not to require the individual's cognitive participation, in the form of special understanding, inference, or explanation. The demonstration of the social facilitation effect across species, from humans to cockroaches, was an indication that the receptivity of the organism to the presence of another may indeed be controlled initially by just such very minimal mechanisms. Sanders' integration, with its emphasis on active, attentive, or cognitively mediated behavior on the part of individual leads researchers to a position where they may ignore a variety of theoretical alternatives before they have been fully examined.

In sum, Sanders' article is useful in keeping alive the social facilitation problem, but I do not think the conclusion that the best direction for future research is to confirm the Attentional Processes model can be justified at this point. Simple hypotheses that do not do an adequate job in explaining the entire phenomenon of social facilitation should not be discarded in favor of larger, more complex hypotheses that are no more adequate. Sanders' article suggests that the goal driving social facilitation research has changed. We are no longer interested in demonstrating just that an organism's dominant response can be enhanced by the presence of a species mate. It can. We are now left with the question of howand with the task of developing a theory of social facilitation. This typc of theory development would be enhanced by a sharp definition of the problem including a set of conditions for identifying the dominant response, a more precise comparison of relevant studies, increased attention to the relationship between various factors and drive induction, and a more complete synthesis of the various theoretical alternatives. A satisfactory synthesis might begin with the most simple explanations of social facilitation and then add factors as they are needed to account for the effect under various task demands and within different social contexts.

\section{REFERENCE}

Zajonc, R. B. Compresence. In P. Paulus (Ed.), Psychology of group influence. Hillsdale, N.J.: Erlbaum. 1980. 